PROCEEDINGS OF THE

AMERICAN MATHEMATICAL SOCIETY

Volume 136, Number 11, November 2008, Pages 3911-3919

S 0002-9939(08)09379-9

Article electronically published on May 20, 2008

\title{
GENERALIZED CAUCHY DIFFERENCE EQUATIONS. II
}

\author{
BRUCE EBANKS
}

(Communicated by David Preiss)

\begin{abstract}
The main result is an improvement of previous results on the equation

$$
f(x)+f(y)-f(x+y)=g[\phi(x)+\phi(y)-\phi(x+y)]
$$

for a given function $\phi$. We find its general solution assuming only continuous differentiability and local nonlinearity of $\phi$. We also provide new results about the more general equation

$$
f(x)+f(y)-f(x+y)=g(H(x, y))
$$

for a given function $H$. Previous uniqueness results required strong regularity assumptions on a particular solution $f_{0}, g_{0}$. Here we weaken the assumptions on $f_{0}, g_{0}$ considerably and find all solutions under slightly stronger regularity assumptions on $H$.
\end{abstract}

\section{INTRODUCTION}

We consider the functional equation

$$
f(x)+f(y)-f(x+y)=g(H(x, y)),
$$

for $x, y \in I$, where $I$ is a real interval such that $I+I \subset I$, where $H: I \times I \rightarrow \mathbb{R}$ is a given function, and $f: I \rightarrow \mathbb{R}$ and $g: H(I \times I) \rightarrow \mathbb{R}$ are unknown functions. Of particular interest is the special case

$$
f(x)+f(y)-f(x+y)=g(\phi(x)+\phi(y)-\phi(x+y)),
$$

where $\phi: I \rightarrow \mathbb{R}$ is a given function.

The results presented here complement and improve earlier results published in [3. As mentioned there, equations of this form such as

$$
f(x+y)-f(x)-f(y)=g(x y)
$$

and

$$
f(x+y)-f(x)-f(y)=f\left(x^{-1}+y^{-1}\right)
$$

have been studied by various authors (see [5, [9, 10, 4, 6, 2]). This led to the question of whether a general method for solving equations of this type might be developed. Another recent related work [8] concerns Cauchy differences which are simultaneously quasi-sums, and this problem also fits the mold of equation (1.1).

Received by the editors June 28, 2006, and, in revised form, September 20, 2007.

2000 Mathematics Subject Classification. Primary 39B22.

Key words and phrases. Cauchy difference, cocycle equation, functional independence, Pexider equation, implicit function theorem, philandering, regularity properties, functional equations.

(C)2008 American Mathematical Society Reverts to public domain 28 years from publication 
We seek nontrivial solutions of (1.1) and (1.2). Observe that for any given $H$ or $\phi$ these equations always admit solutions of the form

$$
g(u)=c, \quad f(x)=A(x)+c,
$$

where $c$ is an arbitrary constant and $A$ is an arbitrary solution of the Cauchy equation

$$
A(x+y)=A(x)+A(y) .
$$

Such an $A$ is called an additive function, and such an $f$ will be called affine. Also observe that if $H$ (or $\phi$ ) is constant, then (1.1) (or (1.2) implies that $f$ is affine. Therefore we call any solution pair $(f, g)$ of (1.1) or (1.2) in which $f$ is affine a trivial solution.

We also observe that in each of the equations (1.3), (1.4), the given function $H$ has the form

$$
H(x, y)=\Psi[\phi(x)+\phi(y)-\phi(x+y)],
$$

assuming that our variables lie in an appropriate domain (the positive reals, for instance). In (1.3) we have $\phi(x)=x^{2}$, while in (1.4) we can take $\phi(x)=\log x$. The question of existence of nontrivial solutions of (1.1) is intimately connected with the representability of $H$ in the form (1.5). That is why equation (1.2) is such an important special case.

In the next section we treat the general equation (1.1), showing that under rather mild conditions it reduces to the special case (1.2). We use "regularityimproving" methods on (1.1); specifically, we give conditions under which a solution pair $(f, g)$ that is measurable or has the Baire property must be twice continuously differentiable. Our main results appear in the third section of the paper. There we present all solutions of (1.2) without any regularity assumptions on the unknown functions.

We close this introduction with some further definitions and notation. We use the notation $C^{k}(D)$ for the class of $k$-times continuously differentiable real functions on a domain $D$. For any function $h: I \rightarrow \mathbb{R}$ we define the associated function $\mathfrak{C} h: I \times I \rightarrow \mathbb{R}$, called the Cauchy difference of $h$, by

$$
\mathfrak{C} h(x, y):=h(x)+h(y)-h(x+y), x, y \in I .
$$

A function $h: D \rightarrow \mathbb{R}$ is called philandering if it is not constant on any nonvoid open subset of $D$. We use the notation $H_{x}, H_{y}$ for the partial derivatives of $H$ with respect to $x$ and $y$ (respectively).

\section{Results of EQUATION (1.1)}

The following appeared as Proposition 10 in 3 .

Theorem 1. Let $I$ be a nonvoid real interval with $I+I \subset I$, and suppose there exists a particular solution $\left(f_{0}, g_{0}\right)$ of (1.1) with $f_{0}, g_{0}$, and $H$ continuously differentiable on their respective domains, and such that the Cauchy difference $\mathfrak{C} f_{0}$ is philandering. Then $g_{0}$ is invertible and $H$ has the form

$$
H=g_{0}^{-1} \circ \mathfrak{C} f_{0} .
$$

Our first aim is to show that we can weaken the regularity assumptions on $f_{0}$ and $g_{0}$ considerably if we strengthen the assumptions on $H$ a bit. 
Proposition 1. Let $I$ be a nonempty interval of $\mathbb{R}$ such that $I+I \subset I$, and suppose $H: I \times I \rightarrow \mathbb{R}$ satisfies the following three conditions: (i) $H \in C^{2}(I \times I)$, (ii) $H_{y}$ is never zero, and (iii) for each $u \in H(I \times I)$ there exists a point $\left(x_{0}, y_{0}\right) \in I \times I$ for which $H\left(x_{0}, y_{0}\right)=u$ and $H_{x}\left(H_{y}-H_{x}\right)\left(x_{0}, y_{0}\right) \neq 0$. Then every solution pair $(f, g)$ of (1.1) that is measurable or possesses the Baire property must be twice continuously differentiable. Additionally, if $H \in C^{k}(I \times I)$ for some $k>2$, then also $f \in C^{k}(I)$ and $g \in C^{k}(H(I \times I))$.

Proof. First, write (1.1) in the form

$$
f(x)=-f(y)+f(x+y)+g(H(x, y)) .
$$

In the case that $f$ and $g$ are measurable we shall apply Theorem 8.3 in [7] to (2.1). Using the notation of that theorem, we take $n=2, Z=Z_{i}=\mathbb{R}(i=0,1,2), T=$ $Y=I, D=I \times I, A_{1}=X_{1}=2 I, A_{2}=X_{2}=H(I \times I) \subset \mathbb{R}, f_{0}=-f, f_{1}=f, f_{2}=$ $g, g_{1}(t, y)=t+y, g_{2}=H$, and $h\left(t, y, u_{0}, u_{1}, u_{2}\right)=-u_{0}+u_{1}+u_{2}$. By assumptions (i) and (ii) on $H$, all hypotheses of the cited Theorem 8.3 are fulfilled, therefore $f$ is continuous.

Next we substitute $u=H(x, y)$ in (2.1) and attempt to solve for $y$ locally as a function of $x$ and $u$. For each such $u$ we select a point $\left(x_{0}, y_{0}\right) \in I \times I$ with the properties in condition (iii) of our theorem. Then we apply the Implicit Function Theorem, as found for example in [11. Condition (ii) on $H$ guarantees the existence of open sets $U \subset \mathbb{R}^{3}, V \subset \mathbb{R}^{2}$ and a map $K: V \rightarrow I$ such that $\left(y_{0}, x_{0}, u\right) \in U,\left(x_{0}, u\right) \in V$ and $y=K(x, u)$ for all $(y, x, u) \in U$. Thus we can write (1.1) locally as

$$
g(u)=f(x)+f(K(x, u))-f(x+K(x, u)),(x, u) \in V,
$$

for some open set $V \subset I \times H(I \times I)$. Once again we apply Theorem 8.3 from [7], this time with $g_{1}(u, x)=K(x, u), g_{2}(u, x)=x+K(x, u)$. Necessary conditions for the application of Theorem 8.3 here are

$$
\frac{\partial g_{1}}{\partial x}=K_{x}=\frac{-H_{x}}{H_{y}} \neq 0, \frac{\partial g_{2}}{\partial x}=1+K_{x}=\frac{H_{y}-H_{x}}{H_{y}} \neq 0
$$

in a neighborhood of the point $\left(u, x_{0}\right)$. But these conditions are guaranteed by our hypothesis (iii) and the continuity of $H_{x}$ and $H_{y}$, hence $g$ is continuous in a neighborhood of $u$. Since $u$ was arbitrary, this means $g$ is continuous on $H(I \times I)$.

In the case that $f$ and $g$ have the Baire property, we proceed in the same manner, but we apply Theorem 9.2 from [7] instead of Theorem 8.3.

Next, supposing that $f$ and $g$ are continuous we apply Theorems 11.3 and 15.2 from [7] to conclude that $f$ and $g$ are two times continuously differentiable. Our final statement follows from repeated application of the cited Theorem 15.2.

We note that the roles of $x$ and $y$ in the partial derivative conditions (ii) and (iii) on $H$ could be reversed. Combining our Proposition 1 with Theorem 1 above, we immediately obtain the following consequence.

Corollary 1. In addition to the hypotheses of the previous proposition, suppose there exists a particular solution $\left(f_{0}, g_{0}\right)$ of (1.1) that is measurable or has the Baire property and such that $f_{0}$ has a philandering Cauchy difference. Then $g_{0}$ is invertible and $H$ has the form

$$
H=g_{0}^{-1} \circ \mathfrak{C} f_{0} .
$$


Observe that if $H$ has the form asserted above, then equation (1.1) reduces to

$$
\mathfrak{C} f(x, y)=g \circ g_{0}^{-1} \circ \mathfrak{C} f_{0}(x, y),
$$

which is equation (1.2) with $g$ replaced by $g \circ g_{0}^{-1}$ and with $\phi=f_{0}$. That is why we devote more attention to equation (1.2) in the next section.

On the other hand, we can sometimes apply Proposition 1 directly, as the next example shows.

Example 1. Suppose $f: I \rightarrow \mathbb{R}$ and $g: 2 I^{2} \rightarrow \mathbb{R}$ satisfy

$$
f(x)+f(y)-f(x+y)=g\left(x^{2}+y^{2}\right), x, y \in I,
$$

where $I=(a, \infty)$ for some $a \geq 0$. (Note that only trivial solutions exist if $0 \in I$.) Let $H(x, y)=x^{2}+y^{2}$. Then $H$ satisfies conditions (i), (ii), (iii) of the proposition, since for each $u \in 2 I^{2}$ there exist $x_{0} \neq y_{0} \in I$ with $u=x_{0}^{2}+y_{0}^{2}$. Hence every measurable solution pair is in $C^{2}$ (actually $C^{\infty}$ ). Differentiating (2.3) with respect to $x$ and $y$, we get

$$
-f^{\prime \prime}(x+y)=4 x y g^{\prime \prime}\left(x^{2}+y^{2}\right), x, y \in I .
$$

Choose $c>2 a$ and put $y=c-x$; then

$$
-f^{\prime \prime}(c)=4 x(c-x) g^{\prime \prime}\left(x^{2}+(c-x)^{2}\right), x \in(a, c-a) .
$$

Letting $u=x^{2}+(c-x)^{2}$, this can be written as

$$
g^{\prime \prime}(u)=\gamma_{c}\left(u-c^{2}\right)^{-1}, u \in\left(c^{2} / 2, a^{2}+(c-a)^{2}\right),
$$

where $\gamma_{c}=f^{\prime \prime}(c) / 2$. Now we can cover $2 I^{2}=\left(2 a^{2}, \infty\right)$ with overlapping intervals of the form $\left(t^{2} / 2, a^{2}+(t-a)^{2}\right)$ for $t \in(2 a, \infty)$. Hence the representation (2.4) extends to all of $2 I^{2}$, and all of the representations must agree. But that means

$$
g^{\prime \prime}(u)=0, u \in 2 I^{2} \text {, and } f^{\prime \prime}(t)=0, t \in 2 I .
$$

Now we substitute

$$
f(t)=A t+B(t \in 2 I), g(u)=C u+D\left(u \in 2 I^{2}\right)
$$

back into (2.3) and find that $C=0, D=B$, and the representation of $f$ extends to all of $I$. Therefore every measurable solution is trivial.

\section{MAIN RESUlTS}

Now we turn our attention to the more special equation (1.2)

$$
f(x)+f(y)-f(x+y)=g(\phi(x)+\phi(y)-\phi(x+y)),
$$

where $\phi$ is given and $f$ and $g$ are unknown. In order to apply the "regularityimproving" results of the proposition in the previous section, we would require (condition (ii))

$$
H_{y}(x, y)=\phi^{\prime}(y)-\phi^{\prime}(x+y) \neq 0, x, y \in I .
$$

This means that any common values of $\phi^{\prime}$ would need to be separated by a distance less than $a$ if $I=(a, \infty)$ for $a>0$, and it would be impossible if $0 \in I$. Thus we see that the results of the previous section will not in general be useful in the special case of equation (1.2). Of course they will apply if $\phi^{\prime}$ is strictly monotonic, in which case $\phi$ is strictly convex or concave, but in that case we have already determined the general solution of (1.2) in [3].

Instead we shall take a new approach. We forego any regularity-improving machinery and instead find the general solution. (From this the regular solutions are 
easily deduced.) We will assume of our given function only that $\phi \in C^{1}(I)$ and $\phi^{\prime}$ is philandering.

Theorem 2. Let $I$ be a real interval for which $I+I \subset I$, assume the given map $\phi: I \rightarrow \mathbb{R}$ is continuously differentiable with philandering derivative, and let $f$ : $I \rightarrow \mathbb{R}, g: \mathfrak{C} \phi(I \times I) \rightarrow \mathbb{R}$ be a solution of (1.2). Then there exist a real constant $c$ and additive maps $A_{1}, A_{2}: \mathbb{R} \rightarrow \mathbb{R}$ such that

$$
f(x)=A_{1}[\phi(x)]+A_{2}(x)+c, g(u)=A_{1}(u)+c,
$$

for all $x \in I, u \in \mathfrak{C} \phi(I \times I)$.

Proof. As in [3] we begin by noting that the left member of (1.2) is a coboundary, hence it satisfies the cocycle equation. Applying the cocycle identity to the right member of (1.2), we have for all $x, y, z \in I$

$$
g(\mathfrak{C} \phi(x, y))+g(\mathfrak{C} \phi(x+y, z))=g(\mathfrak{C} \phi(x, y+z))+g(\mathfrak{C} \phi(y, z)) .
$$

Let $T: I \times I \times I \rightarrow \mathbb{R}$ be the transformation $(x, y, z) \longmapsto(u, v, w)$ defined by

$$
\begin{aligned}
u & =u(x, y, z):=\mathfrak{C} \phi(x, y), \\
v & =v(x, y, z):=\mathfrak{C} \phi(x+y, z), \\
w & =w(x, y, z):=\mathfrak{C} \phi(y, z) .
\end{aligned}
$$

We can rewrite the previous equation as

$$
g(u)+g(v)=g(u+v-w)+g(w) .
$$

Our aim is to show that $u, v, w$ can be treated as independent variables in this equation, at least locally.

Suppose the Jacobian determinant of $T$ were equal to zero on some open set $O \subset I \times I \times I$ having nonempty intersection with the plane $x=y$. Then $u, v, w$ would be functionally dependent on $O$. On the other hand,

$$
\frac{\partial(u, v)}{\partial(x, y)}=\left[\phi^{\prime}(x)-\phi^{\prime}(y)\right]\left[\phi^{\prime}(x+y)-\phi^{\prime}(x+y+z)\right] .
$$

This expression cannot be identically zero on any nonvoid open set since $\phi^{\prime}$ is philandering, hence $u, v$ are functionally independent on $O$. Thus there would exist a function $\sigma$ such that $w=\sigma(u, v)$, that is,

$$
\mathfrak{C} \phi(y, z)=\sigma[\mathfrak{C} \phi(x, y), \mathfrak{C} \phi(x+y, z)]
$$

on $O$. Observe that the right member of this equation is symmetric in $x$ and $y$. Interchanging $x$ and $y$ we get $\mathfrak{C} \phi(y, z)=\mathfrak{C} \phi(x, z)$, or

$$
\phi(y)-\phi(y+z)=\phi(x)-\phi(x+z)
$$

for all $(x, y, z) \in O$ such that $(y, x, z) \in O$. Therefore there would be an open set on which $\phi$ is affine, but this is impossible since $\phi^{\prime}$ is philandering.

Therefore there exist points $(x, y, z)$ such that the Jacobian of $T$ is nonzero; indeed, such points exist in every open set intersecting the plane $x=y$. Since $\phi^{\prime}$ is continuous, the set

$$
N=\left\{(x, y, z) \in I \times I \times I: \frac{\partial(u, v, w)}{\partial(x, y, z)} \neq 0\right\}
$$


is a nonempty open set, as is $T(N)$ by the Open Mapping Theorem. Thus we have (3.4) for $(u, v, w)$ in an open set $T(N)$. Let $U$ be a connected component of $T(N)$ and write (3.4) in the form

$$
g(u)+g(v)=h(u+v)
$$

for $(u, v) \in U^{\prime}=\{(u, v): \exists w$ with $(u, v, w) \in U\}$. (Recall that $w$ is functionally independent of $u$ and $v$.) Now (see e.g. pp. 76-80 in [1]) such Pexider equations have unique extensions to the whole plane $\mathbb{R}^{2}$; hence

$$
g(u)=A_{1}(u)+c_{0}, u \in \mathfrak{C} \phi(D),
$$

for some open connected set $D \subset I \times I$, some constant $c_{0}$, and some additive map $A_{1}: \mathbb{R} \rightarrow \mathbb{R}$.

Let $J \times K$ be an open rectangle contained in $D$. Note that $\mathfrak{C} \phi(J \times K)=\mathfrak{C} \phi(K \times J)$ by the symmetry of $\mathfrak{C} \phi$. We define for any two sets $S_{1}, S_{2}$,

$$
\mathfrak{C} \phi\left(S_{1}, S_{2}\right):=\left\{\mathfrak{C} \phi(x, y): x \in S_{1}, y \in S_{2}\right\}=\mathfrak{C} \phi\left(S_{1} \times S_{2}\right)=\mathfrak{C} \phi\left(S_{2} \times S_{1}\right),
$$

and from above write

$$
g(u)=A_{1}(u)+c_{0}, u \in \mathfrak{C} \phi(J, K) .
$$

Substituting $(x, y) \in J \times K$ into (1.2) yields

$$
f(x)+f(y)-f(x+y)=A_{1}[\phi(x)+\phi(y)-\phi(x+y)]+c_{0} .
$$

Thus the map $\psi: J \cup K \cup(J+K) \rightarrow \mathbb{R}$ defined by

$$
\psi(x):=f(x)-A_{1}[\phi(x)]-c_{0}, x \in J \cup K \cup(J+K)
$$

satisfies the Cauchy equation

$$
\psi(x)+\psi(y)=\psi(x+y),(x, y) \in J \times K .
$$

Any such function has a "quasi-extension" to $\mathbb{R}$ such that

$$
\psi(x)=A_{2}(x)+\left\{\begin{array}{c}
a_{0} \text { if } x \in J, \\
b_{0} \text { if } x \in K, \\
a_{0}+b_{0} \text { if } x \in J+K
\end{array}\right.
$$

for some additive map $A_{2}: \mathbb{R} \rightarrow \mathbb{R}$ and constants $a_{0}, b_{0}$. So $f$ has the representation

$$
f(x)=A_{1}[\phi(x)]+\psi(x)+c_{0}, x \in J \cup K \cup(J+K)
$$

in terms of such a function $\psi$. Now we try to extend these representations (3.6), (3.5) for $f, g$, respectively, to the sets

$$
\bigcup_{n=0}^{\infty}(n J+K) \cup J, \mathfrak{C} \phi\left(\bigcup_{n=0}^{\infty}(n J+K), J\right) .
$$

To simplify notation, let $J_{n}=n J+K$ for $n=1,2,3, \ldots$. Note that $J_{0}=K$ and $J_{n}+J=J_{n+1}$ for all $n \geq 0$. Also, for any intervals $L, M$ and real numbers $a_{0}, b_{0}$ define the "Pexider step function" $P\left(L, M, a_{0}, b_{0}\right)$ on $L \cup M \cup(L+M)$ by

$$
P\left(L, M, a_{0}, b_{0}\right)(x)=\left\{\begin{array}{c}
a_{0} \text { if } x \in L, \\
b_{0} \text { if } x \in M, \\
a_{0}+b_{0} \text { if } x \in L+M .
\end{array}\right.
$$


Suppose we have shown for some $n \geq 0$ that

$$
\begin{aligned}
& g(u)=A_{1}(u)+c_{n}, \quad u \in \mathfrak{C} \phi\left(J, \bigcup_{i=0}^{n} J_{i}\right), \\
& f(x)=A_{1}[\phi(x)]+A_{2}(x)+c_{k}+P\left(J, J_{k}, a_{k}, b_{k}\right)(x), x \in J \cup J_{k} \cup J_{k+1}(k=0, \ldots, n) .
\end{aligned}
$$

(For $n=0$ these are equations (3.6), (3.5).) In (3.2) letting $x \in J, y \in J_{n}, z \in J$, we have

$$
A_{1}[\mathfrak{C} \phi(x, y)]+g(\mathfrak{C} \phi(x+y, z))=g(\mathfrak{C} \phi(x, y+z))+A_{1}[\mathfrak{C} \phi(y, z)]
$$

by the inductive hypothesis. Defining variables $s, t$ by

$$
s=\mathfrak{C} \phi(x+y, z), t=\mathfrak{C} \phi(x, y+z)
$$

and rearranging, we get

$$
g(s)-A_{1}(s)=g(t)-A_{1}(t) .
$$

The Jacobian of the transformation $(x, y) \mapsto(s, t)$ on $J \times J_{n}$ is given by

$$
\frac{\partial(s, t)}{\partial(x, y)}=\left[\phi^{\prime}(x+y)-\phi^{\prime}(x+y+z)\right]\left[\phi^{\prime}(y+z)-\phi^{\prime}(x)\right] .
$$

We claim that this Jacobian cannot be zero on any open set since $\phi^{\prime}$ is philandering. Indeed, choose $\left(x_{0}, y_{0}, z_{0}\right) \in J \times J_{n} \times J$ such that $\phi^{\prime}\left(x_{0}+y_{0}\right)-\phi^{\prime}\left(x_{0}+y_{0}+z_{0}\right) \neq 0$. Then since $\phi^{\prime}$ is continuous there exists an open set $V$ containing $\left(x_{0}, y_{0}, z_{0}\right)$ in which $\phi^{\prime}(x+y)-\phi^{\prime}(x+y+z) \neq 0$. But it cannot happen that $\phi^{\prime}(y+z)-\phi^{\prime}(x)=0$ identically in $V$ since $\phi^{\prime}$ is philandering. Hence $s$ and $t$ are functionally independent on $J \times J_{n}$ and we deduce from (3.7) that

$$
g(u)=A_{1}(u)+c_{n+1}, u \in \mathfrak{C} \phi\left(J, J_{n+1}\right),
$$

for some constant $c_{n+1}$. As before we obtain from (1.2) that for some additive map $A_{3}: \mathbb{R} \rightarrow \mathbb{R}$ and real constants $a_{n+1}, b_{n+1}$ our function $f$ has the form

$f(x)=A_{1}[\phi(x)]+A_{3}(x)+c_{n+1}+P\left(J, J_{n+1}, a_{n+1}, b_{n+1}\right)(x), x \in J \cup J_{n+1} \cup J_{n+2}$.

By the inductive hypothesis we know that $f$ has the form $f(x)=A_{1}[\phi(x)]+A_{2}(x)+$ $c_{n}+P\left(J, J_{n}, a_{n}, b_{n}\right)(x)$ for $x \in J \cup J_{n} \cup J_{n+1}$. Comparing these forms, we see that

$$
\begin{aligned}
& A_{3}(x)+c_{n+1}+a_{n+1}=A_{2}(x)+c_{n}+a_{n} \text { for } x \in J, \\
& A_{3}(x)+c_{n+1}+b_{n+1}=A_{2}(x)+c_{n}+a_{n}+b_{n} \text { for } x \in J_{n+1} .
\end{aligned}
$$

Thus the additive functions $A_{3}$ and $A_{2}$ must be identical on $\mathbb{R}$ and the asserted forms of $f, g$ are established for all $n$.

Next, we claim that $\bigcup_{i=0}^{\infty} J_{i}$ contains an interval of the form $(M, \infty)$ for sufficiently large $M$ (or an interval of the form $(-\infty,-M)$ if $I$ is of the form $(-\infty,-a)$ or $(-\infty,-a])$. Let $J=(\gamma, \delta), K=(\rho, \tau)$; then $J_{n} \cap J_{n+1} \neq \emptyset$ as soon as $n(\delta-\gamma)>$ $\gamma+\rho-\tau$. It follows that $J_{k} \cap J_{k+1} \neq \emptyset$ for all $k \geq N$ for some positive integer $N$. Hence

$$
a_{k}=0, c_{k}+b_{k}=c_{k+1}+b_{k+1} \text {, for all } k \geq N .
$$

Therefore, defining $c$ to be the common value of $c_{k}+b_{k}$ for all $k \geq N$, we have

$$
f(x)=A_{1}[\phi(x)]+A_{2}(x)+c, x \in(M, \infty) .
$$

Inserting this back into (1.2) we see that

$$
A_{1}[\phi(x)+\phi(y)-\phi(x+y)]+c=g(\phi(x)+\phi(y)-\phi(x+y))
$$


for all $x, y \in(M, \infty)$. Finally, by Theorem 23 in [3] these forms of $f$ and $g$ extend to their whole domains, and the proof is complete.

Note that in [3] we achieved this result only under quite strong assumptions on $\phi$, namely analyticity or strict convexity. Now we have much weaker assumptions about $\phi$, and this settles a question posed in [3].

Remark 1. As in [3], the results above can be carried over to generalized Cauchy differences in which addition is replaced by a quasi-sum. That is, we can also treat functional equations of the form

$$
f(s)+f(t)-f \circ \theta^{-1}(\theta(s)+\theta(t))=g(H(s, t))
$$

and, in particular,

$$
f(s)+f(t)-f \circ \theta^{-1}(\theta(s)+\theta(t))=g\left[\phi(s)+\phi(t)-\phi \circ \theta^{-1}(\theta(s)+\theta(t))\right],
$$

where $\theta$ is an invertible function satisfying appropriate conditions.

For example, take $I=(0, \infty)$ and let $\theta: I \rightarrow \mathbb{R}$ be defined by $\theta(x)=\log x$. Then the general solution of the equation

$$
f(s)+f(t)-f(s t)=g[\phi(s)+\phi(t)-\phi(s t)],
$$

for $s, t \in I$, is given by

$$
f(t)=A[\phi(t)]+L(t)+c, g(u)=A(u)+c,
$$

under the hypotheses that the function $x \longmapsto \phi\left(\theta^{-1}(x)\right)=\phi\left(e^{x}\right)$ is continuously differentiable and locally nonlinear. Here $c$ is an arbitrary constant, $A: \mathbb{R} \rightarrow \mathbb{R}$ is an arbitrary additive map, and $L: I \rightarrow \mathbb{R}$ is an arbitrary solution of Cauchy's logarithmic functional equation $L(x y)=L(x)+L(y)$. (Here $L=A_{2} \circ \log$.)

\section{REFERENCES}

[1] J. Aczél, A Short Course on Functional Equations Based upon Recent Applications to the Social and Behavioral Sciences, Reidel/Kluwer, Dordrecht/Boston, 1987. MR875412 (88d:39013)

[2] B. R. Ebanks, On Heuvers' logarithmic functional equation, Results Math. 42 (2002), 37-41. MR.1934223 (2003j:39070)

[3] B. R. Ebanks, Generalized Cauchy difference functional equations, Aequationes Math. 70 (2005), 154-176. MR2167992 (2006d:39040)

[4] B. R. Ebanks, P. L. Kannappan, and P. K. Sahoo, Cauchy differences that depend on the product of arguments, Glas. Mat. Ser. III 27(47) (1992), 251-261. MR.1244642 (94k:39045)

[5] I. Ecsedi, On the functional equation $f(x+y)-f(x)-f(y)=g(x y)$, Mat. Lapok 21 (1970), 369-374 (1971). MR0306754 (46:5876)

[6] K. J. Heuvers, Another logarithmic functional equation, Aequationes Math. 58 (1999), 260264. MR.1715396 (2001a:39055)

[7] A. Járai, Regularity Properties of Functional Equations in Several Variables, Springer, New York, 2005. MR2130441 (2006b:39001)

[8] A. Járai, Gy. Maksa, and Zs. Páles, On Cauchy-differences that are also quasisums, Publ. Math. Debrecen 65 (2004), 381-398. MR2107955 (2005h:39042)

[9] K. Lajkó, Special multiplicative deviations, Publ. Math. Debrecen 21 (1974), 39-45. MR0364932 (51:1186) 
[10] Gy. Maksa, On the functional equation $f(x+y)+g(x y)=h(x)+h(y)$, Publ. Math. Debrecen 24 (1977), 25-29. MR0447867(56:6177)

[11] W. Rudin, Principles of Mathematical Analysis, McGraw Hill, New York, 1976. MR 0385023 $(52: 5893)$

Department of Mathematics and Statistics, P.O. Box MA, Mississippi State UniverSity, Mississippi State, Mississippi 39762

E-mail address: ebanks@math.msstate.edu 